\title{
Ultraschall in der Altersmedizin: Schwere Verläufe bei COVID-19 frühzeitig aufspüren; einem Schlaganfall und dem lebensbedrohlichen Bauchaortenaneurysma vorbeugen
}

\author{
Online-Pressekonferenz der Deutschen Gesellschaft für Ultraschall in der Medizin

\section{e. V. (DEGUM) am 1. Dezember 2020}

Der Ultraschall als schonende und einfach anzuwendende Methode der Bildgebung ist gerade in der Altersmedizin vielseitig einsetzbar, gilt in vielen Bereichen als wichtige Vorsorgeuntersuchung und kann frühzeitig lebensbedrohliche Situationen verhindern. So hat die DEGUM am 1. Dezember 2020 eine Online-Pressekonferenz zum Thema „Ultraschall in der Altersmedizin: Schwere Verläufe bei COVID-19 frühzeitig aufspüren; einem Schlaganfall und dem lebensbedrohlichen Bauchaortenaneurysma vorbeugen " veranstaltet. 38 Journalisten haben an der Pressekonferenz teilgenommen. Auf der DEGUM-Webseite finden Interessierte den Video-Mitschnitt der PK.

\section{COVID-19-Komplikationen am Herzen mit Ultraschall schnell und sicher erkennen}

Vor allem viele ältere Patienten entwickeln im Laufe einer COVID-19-Erkrankung Komplikationen am Herzen, beispielsweise eine Herzmuskelentzündung. Langzeitfolgen wie Luftnot, Müdigkeit und Abgeschlagenheit, unter denen viele COVID-Patienten leiden, können auch auf eine durch die Infektion entstandene Herzschwäche hindeuten. Insbesondere ältere Patienten mit kardialen Vorerkrankungen scheinen ein erhöhtes Risiko für schwere Verläufe von COVID-19 zu haben. Umso wichtiger ist es, Anzeichen für Schäden am Herzen früh zu erkennen. Die schnellste und einfachste Methode ist dabei die Echokardiografie. Wie genau der
Herzultraschall bei akuter Infektion und in der Nachsorge eingesetzt wird, erklärte Professor Dr. med. Fabian Knebel, Leitender Oberarzt an der Berliner Charité, Klinik für Kardiologie am Campus Mitte und Kursleiter und Arbeitskreisleiter Echokardiografie in der DEGUM, auf der Online-Pressekonferenz.

Die Echokardiografie, die Ultraschalluntersuchung des Herzens, ist eine der wichtigsten Routineuntersuchungen am Herzen. Diese Ultraschalluntersuchung nutzt der Kardiologe, um zum Beispiel eine Herzinsuffizienz zu erkennen oder den Zustand der Herzklappen zu beurteilen. Die transösophageale Echokardiografie (auch Schluckecho genannt), bei der eine Ultraschallsonde über die Speiseröhre eingeführt wird, liefert Erkenntnisse über Blutgerinnsel in den Vorhöfen oder, bei Verdacht, auf eine bakterielle Infektion der Herzklappen (Endokarditis). „Die Echokardiografie ist die zentrale Bildgebung in der Kardiologie und in der Altersmedizin nicht wegzudenken“, erklärt Professor Dr. med. Fabian Knebel. Sie sei vielseitig einsetzbar, habe keine Kontraindikation, geringe Kosten und könne auch für Wiederholungsuntersuchungen genutzt werden, so der Leiter des Arbeitskreises Echokardiografie in der DEGUM.

Vor allem aber auch in Zeiten der COVID-19Pandemie hat sich der Herzultraschall zu einem wichtigen Instrument entwickelt [1]. „Insbesondere ältere Patienten mit kardialen Vorerkrankungen haben ein erhöhtes Risiko für schwere Verläufe von COVID-19“, sagt Knebel. Wer beispielsweise an Störungen der linksventrikulären Pumpfunktion leidet oder einen Herzklappenfehler hat, sollte sich im Falle einer COVID-19-Infektion dringend einer Echokardiografie unterziehen. Dies gelte auch für Patienten ohne bekannte Vorerkrankungen. „In der Corona-Pandemie hat sich gezeigt, dass eine engmaschige echokardiografische Untersuchung von COVID-Patienten sinnvoll ist“", betont Knebel und verweist auf die erhöhte Gefahr für eine Herzmuskelentzündung (Myokarditis) während einer Infektion. „Hier bietet es sich an, moderne echokardiografische Methoden wie den Screen einzusetzen." Dies belegen auch Studien.

Laut einer Publikation in der Fachzeitschrift Jama Cardiology [2] hatten von 100 Patienten, die durch das hessische COVID-19Testzentrum identifiziert wurden, Wochen nach der Genesung 78 Auffälligkeiten am Herzen - davon wiesen 60 Anzeichen einer Herzmuskelentzündung auf. Hier wurde das Herz mittels MRT untersucht. Eine frühe Diagnose sei deshalb wichtig, da die rechtzeitige Behandlung schwere Entzündungsschäden verringern oder sogar aufhalten kann. „Wir empfehlen in jedem Fall, dass Patienten, die nach einer COVID-19-Infektion beispielsweise weiterhin an Luftnot oder Abgeschlagenheit leiden, sich echokardiografisch untersuchen lassen, um neben einer Myokarditis auch eine Verminderung der Herzfunktion zu erkennen“, sagt Knebel.

Immer mehr Studien deuten darauf hin, dass es sich bei COVID-19 um eine Erkrankung des Endothels handelt [3]. Die Schädi- 
gung des Endothels durch SARS-CoV-2 bildet den Beginn einer Reaktionskette, die mit der Thrombosierung der kleinen Blutgefäße endet. Die Folge ist eine verstärkte Verklumpungsneigung des Blutes. In Autopsien verstorbener Patienten stellten Mediziner überraschend viele Thrombosen und Blutgerinnsel in Arterien, Venen und auch Kapillaren fest. „Etwa ein Drittel der schwer an COVID-19 erkrankten Patienten verstirbt an Thrombosen und Lungenembolien“, erklärt Knebel. Des Weiteren sei beobachtet worden, dass es bei einigen COVID-Patienten durch Thrombosen und Thromboembolien zu einer Erhöhung des Lungendrucks und einer Vergrößerung des rechten Herzens kommen kann. „Auch hier ist die Echokardiografie eine sehr elegante Methode, um dies frühzeitig und sicher zu detektieren.“

\section{Sonografie beim Bauchaorten- aneurysma kann Leben retten}

Bis zu 2000 Menschen sterben pro Jahr an einer Ruptur, also einem Zerreißen oder Aufplatzen, eines Bauchaortenaneurysmas. In Deutschland gehört die Ultraschalluntersuchung der Bauchaorta für Männer zum durch die Krankenkassen bezahlten Gesundheits-Check-up ab 65 Jahren. Warum zusätzlich eine flächendeckende Einladung zum Screening sinnvoll ist und warum die Vorsorgeuntersuchung auch für Frauen wichtig ist, war ebenfalls Thema der Online-Pressekonferenz.

Schmerzfrei, risikolos und ohne Nebenwirkungen - das ist eine Ultraschalluntersuchung. Sie steht im starken Kontrast zu dem, was sie, im Falle der Ruptur eines Bauchaortenaneurysmas, verhindern kann: Nur $10 \%$ aller Patienten, die eine Ruptur erleiden, erreichen lebend das Krankenhaus, alle anderen versterben bereits auf dem Weg dorthin oder noch zu Hause. Geschätzt 250000 Menschen in Deutschland sind an einem Bauchaortenaneurysma erkrankt.

Das Bauchaortenaneurysma (BAA) ist eine Erweiterung der Bauchschlagader (Aorta) auf einen Durchmesser von mindestens 30 Millimeter. Durch die Ausdünnung der Wand erhöht sich die Rupturgefahr in Ab- hängigkeit des Aneurysmadurchmessers. „Wichtige Faktoren für die Entstehung eines Aneurysmas sind das männliche Geschlecht ab dem 65. Lebensjahr, familiäre Häufung und Nikotinkonsum“, erklärt Dr. med. Siegfried Krishnabhakdi, Chefarzt der Klinik für Gefäßchirurgie, vaskuläreund endovaskuläre Chirurgie im Klinikum Osnabrück GmbH. „Bauchaortenaneurysmen sind in der Bevölkerung nicht selten. Ein Großteil der Aneurysmen wird nach der Diagnose lediglich regelmäßig kontrolliert, nur bei wenigen ist ein Eingriff als Reaktion auf die Diagnose notwendig“, so Krishnabhakdi. Die Ultraschalluntersuchung liefert bei der Diagnosestellung sehr genaue $\mathrm{Er}$ gebnisse. „In beinahe $100 \%$ der Untersuchungen kann die Größe des Aneurysmas zuverlässig bestimmt werden“, sagt der DEGUM-Experte.

Die Kriterien, ab wann eine Kontrolle oder eine Therapie notwendig ist, sind definiert. Ist bei Männern das BAA größer als 55 Millimeter und bei Frauen größer als 50 Millimeter, wird empfohlen, das Aneurysma auszuschalten. Kleinere Aneurysmen mit geringer Wachstumsgeschwindigkeit werden mit Ultraschall weiter beobachtet. Die Intervalle hängen dabei von der Größe, den Risikofaktoren, wie z. B. Nikotinkonsum, und der Wachstumsgeschwindigkeit ab. Da Frauen und noch mehr rauchende Frauen - zur Ruptur neigen, werden hier kürzere Intervalle empfohlen.

Seit 2018 ist das Ultraschallscreening für Männer ab 65 Jahre Teil der Vorsorgemaßnahmen, die von den Krankenkassen bezahlt werden. Die Untersuchung wird von Hausärzten, Urologen, Internisten, Radiologen und Chirurgen durchgeführt. „Die Raten von Gesamtsterblichkeit, Aneurysmabedingter Mortalität, Zahl der Rupturen und Notfalloperationen gingen im Langzeitverlauf durch das Screening signifikant zurück“, betont Krishnabhakdi. Allerdings werde eine Risikogruppe vernachlässigt: die Frauen. „Bei Frauen über 65 Jahren, die rauchen, ist das Risiko der Ruptur eines Bauchaortenaneurysmas sogar höher als bei gleichaltrigen Männern.“ Für Krishnabhakdi wäre deshalb eine Ausweitung des Vorsorge-Screenings auf Frauen über 65 Jahre, die Risikofaktoren wie Nikotinkonsum und Bluthochdruck erfüllen, sinnvoll.

\section{Frühes Ultraschallscreening der Halsschlagadern kann Schlaganfallrisiko verringern}

Rund 265000 Menschen erleiden jährlich in Deutschland einen Schlaganfall. Bei etwa 30000 Patienten ist die Ursache eine Verengung oder ein Verschluss der inneren Halsschlagader - die sogenannte Karotisstenose. Kalkablagerungen in der Carotis können aufbrechen, als Gerinnsel ins Gehirn verschleppt werden und so einen Schlaganfall auslösen. Ultraschall-Experten können heute schon frühzeitig leichte Gefäßveränderungen bis hin zu Carotisstenosen sehr leicht durch eine Sonografie der Halsgefäße diagnostizieren. Ob ein Carotis-Ultraschallscreening im Rahmen von Vorsorgeuntersuchungen sinnvoll ist und ob asymptomatische Carotisstenosen bei Menschen höheren Alters zwingend eine Operation zur Folge haben müssen, erklärte Professor Dr. med. Felix Schlachetzki, Chefarzt am Zentrum für Vaskuläre Neurologie und Intensivmedizin, Klinik und Poliklinik für Neurologie der Universität Regensburg, medbo Bezirksklinikum Regensburg, auf der Online-Pressekonferenz.

Der Schlaganfall gehört nach wie vor zu den häufigsten Todesursachen in Deutschland. Je älter die Patienten sind, desto höher das Risiko. „Die Ultraschalluntersuchung der Halsschlagader ist eine breit verfügbare Technik, um das individuelle Risiko für kardiovaskuläre Ereignisse, insbesondere für Schlaganfälle, abzuschätzen“, erklärt Schlachetzki.

Im Ultraschall kann ein Arzt feststellen, ob die Karotis eine erhöhte Intima-MediaDicke (Verdickung der inneren und mittleren Schicht der Gefäßwand) oder Plaques (Lipid- und Kalkablagerungen) aufweist. „Ist dies der Fall, sollten Patienten dazu ermutigt werden, sich mehr zu bewegen, Übergewicht zu reduzieren sowie gegebenenfalls ihre Ernährung umzustellen und das Rauchen aufzugeben“, so der stellvertretende DEGUM-Arbeitskreisleiter „Vaskulärer Ultraschall“. Außerdem müssen bestehende Risikofaktoren wie Bluthochdruck, Diabetes und Cholesterin konsequent behandelt werden, um das Risiko für einen Gefäßverschluss einzudämmen. Diese prä- 
ventiven Therapien - auch als „Best-medical-treatment“ bezeichnet - seien bei Risikopatienten mit erblicher Vorbelastung auch schon im berufsfähigen Alter unbedingt empfehlenswert. Im Rahmen einer Vorsorgeuntersuchung sei das Karotis-Ultraschallscreening daher durchaus sinnvoll, gibt der Ultraschall-Experte zu Bedenken.

Entstehen durch Kalkablagerungen aus Plaques erst einmal manifeste Stenosen also Engstellen an der Halsschlagader können diese aufbrechen, als Gerinnsel ins Gehirn verschleppt werden und so einen Schlaganfall auslösen. Welche Therapie nach einer entsprechenden Diagnose erfolgen soll, muss interdisziplinär bewertet werden. Das Carotis-Screening darf hier nicht alleinige Entscheidungsgrundlage sein. „Bei allen Patienten mit Stenosen der Halsschlagadern ist eine detaillierte Nutzen-Risiko-Abwägung zwischen den Möglichkeiten der modernen Pharmakotherapie und den operativen Möglichkeiten inklusive Stenting nötig, und da ist auch das reelle Patientenalter ein wichtiges Kriterium“, mahnt der Neurologe. So sind Carotisstenosen, die keine Symptome verursachen, zwar ein Maßstab für Erkrankungen des gesamten arteriellen Systems. „Sie sind aber gleichzeitig nur ein Teil des komplexen arteriellen Hirnversorgungssystems, welches durchaus in der Lage ist, sich anzupassen“, erklärt Schlachetzki. Das führe dazu, dass der Anteil der Arteriosklerose-bedingten Schlaganfälle ab der siebten Lebensdekade wieder sinke. Eine Carotisstenose müsse in diesem Alter deshalb immer in der Zusammenschau des gesamten Gefäßsystemzu- standes betrachtet werden. Zudem seien die möglichen Komplikationsraten einer Carotis-Operation beziehungsweise eines Carotis-Stents gegenüber den Möglichkeiten des „Best-medical-treatment“, von dem auch die Herzinfarkte und arterielle Verschlusskrankheit der Beine profitieren, abzuwägen.

Insgesamt ist aus neurologischer Sicht ein Ultraschallscreening der Halsschlagadern wichtig, um frühe Gefäßwandveränderungen zwischen dem 30. und 70. Lebensjahr an den Karotiden zu erkennen. „Damit können wir das sogenannte Gefäßalter eines Menschen definieren und gegebenenfalls notwendige Lebensveränderungen initiieren“, fasst Schlachetzki zusammen. Hier hilft ein breites Screening bei versierten Ultraschallern. Werden hämodynamisch relevante Stenosen erkannt, sollten jedoch Aktionismus vermieden und die Patienten zu neurovaskulären Experten überwiesen werden. Dazu gehören insbesondere Neurologen oder Angiologen, die auch intrakranielle Gefäße beurteilen können. Eine Operation oder Stent-Therapie ohne vorherige Begutachtung durch diese Spezialisten sei strikt abzulehnen und für den Patienten gefährlich.

\section{Literatur zu Herzkomplikationen am Her- zen:}

[1] Szekely Y, Lichter Y, Taieb P et al. Spectrum of Cardiac Manifestations in COVID-19. A Systematic Echocardiographic Study. Circulation 2020; 142: 342-353
[2] Puntmann VO, Carerj VL, Wieters I et al. Outcomes of Cardiovascular Magnetic Resonance Imaging in Patients Recently Recovered From Coronavirus Disease 2019 (COVID-19). JAMA Cardiol 2020; 5: 12651273

[3] Libby P, Lüscher T. COVID-19 is, in the end, an endothelial disease. Eur Heart J 2020; 41: 3038-3044

\section{Weiterführende Literatur zum Carotis- Screening:}

[1] Nezu T, Hosomi N. Usefulness of Carotid Ultrasonography for Risk Stratification of Cerebral and Cardiovascular Disease. Atheroscler Thromb 2020; 27: 1023-1035

[2] Näslund U, Ng N, Lundgren A et al. Visualization of asymptomatic atherosclerotic disease for optimum cardiovascular prevention (VIPVIZA): a pragmatic, open-label, randomised controlled trial. Lancet 2019; 393: 133-142

[3] Paraskevas KI, Veith FJ, Spence JD. How to identify which patients with asymptomatic carotid stenosis could benefit from endarterectomy or stenting. Stroke Vasc Neurol 2018; 3: 92-100

[4] Abbott A, Brunser AM, Giannoukas A et al. Misconceptions regarding the adequacy of best medical intervention alone for asymptomatic carotid stenosis. J Vasc Surg 2020; 71: 257-269

[5] Keyhani S, Cheng EM, Hoggatt KJ et al. Comparative Effectiveness of Carotid Endarterectomy vs Initial Medical Therapy in Patients With Asymptomatic Carotid Stenosis. JAMA Neurol 2020; 77: 1110-1121 